\title{
Mobility management in IEEE 802.11 WLAN using SDN/NFV technologies
}

\author{
Syed Mushhad M. Gilani, , Tang Hong, Wenqiang Jin, Guofeng Zhao, H. Meng Heang and Chuan Xu
}

\begin{abstract}
IEEE 802.11 wireless LAN is proliferating since the increased trend in the wireless network utilization on mobile devices. Accuracy, fast content delivery, and reliable mobility support are essential features of any network to support the changing trend in a wireless network. However, traditional architectures in wireless LAN (WLANs or WiFi) always endured from challenges such as the provision of consistent mobility, real-time packet flow, and seamless handoff. Generally, most of the WLAN only relies on signal strength for handoff which is not sufficient enough for fair selection of an access point (AP) and therefore causes imperfect performance of the network. We present a novel mobility management scheme for WLANs to deal with the mobility management issues, and load balancing by software-defined network (SDN) and network function virtualization (NFV) technologies. The proposed scheme is based on logical AP ( $A P$ ) that keeps a connection with the user/mobile terminal (MT) during handoff triggered by either the user or the SDN controller for seamless mobility. It also involves the current state of each AP in addition to traditional parameters of WLAN. We implemented the proposed scheme on a real testbed in a WLAN environment. The evaluation results authenticate that our proposed scheme provides robust handover without throughput degradation and load imbalance among adjacent APs, and allocates the best AP in the neighboring region. Moreover, our proposed scheme is feasible to implement since it did not require any modification at the mobile terminal.
\end{abstract}

Keywords: Software-defined network (SDN), Network function virtualization (NFV), Access point (AP), Mobility management, WLAN, Seamless handover, Load balancing

\section{Introduction}

The rapid growth in mobile Internet applications and innovation of mobility services, location-based services, WiFi calling and cloud services, and the IEEE 802.11-based wireless LANs (WLANs/WiFi) has gained a lot of attention in recent years, because the WLAN provides economical solution to support the Internet access services with high throughput in contrast to cellular networks. According to the Juniper Research report [1], the volume of data traffic generated by smart mobile devices will hit nearly 197,000 petabytes (PB) by 2019 that mainly offloaded to WiFi. Meanwhile, some mobile operators also have begun the deployment of WiFi hotspots in high-density areas to overwhelm the threat of LTE spectrum that is near to Shannon's capacity limit [2].

\footnotetext{
* Correspondence: mushhad@hotmail.com

Research Center of Future Internet (RFI), Chongqing University of Posts and Telecommunications, Chongqing 400065, China
}

The proliferation of WiFi consumers has raised some new requirements such as seamless handover, quality of service (QoS), high throughput, load balancing, and ubiquitous network access.

Unfortunately, the existing WiFi infrastructure is unstructured which could lead to the prevailing issues in terms of mobility management. There are some prominent issues: (1) The current WLAN structure is tightly coupled between the control and data plane that restricts the integration of innovative network applications, services, and policies [3]. Thus, it makes the intelligent mobility management functions difficult to implement according to the real-time network state that is required for future network state perception. (2) The traditional IEEE 802.11 APs do not have any built-in feature for centralized network management [4]. Thus, users need to pass through an authentication procedure during handover among APs, which is a time-limited task. In the case of timeout, the network connection will be lost. 
(3) The deployments of APs in a closed region for overwhelming the signal gaps among APs, the purpose is to provide a perfect and continuous connection to the user during mobility. However, the deployment of APs in an adjacent region is caused by signal interference [5]. Using the IEEE 802.11 standard [4], MTs scanned multiple APs within the radio range and received an extensive list of SSIDs. To establish a connection, the user must select a SSID, most probably SSID selection is solely based on the strongest received signal strength indication (RSSI). In the case of association with already congested AP, the user could experience low throughput. The IEEE 802.11 standard [4] did not provide any load balancing mechanism that makes a comprehensive imbalanced network, and therefore, network confronted throughput degradation at the cost of packet loss ratio. The aforementioned issues restrict the perfect utilization of WiFi to fulfill the requirements of the future Internet which results in an inefficient mobility management.

The concept of software-defined network (SDN) is a promising solution in the wireless network personification that is able to provide programmable control plane and data plane into AP. These features boost the WLAN performance regarding the finegrained packet control and provide a configuration interface using the OpenFlow [6]. The OpenFlowbased switch can perform various jobs, defined by the controller based on predefined rules, e.g., it can act as a router, firewall, NAT [7], or other userdefined functions. The OpenFlow may incorporate handover parameters in existing applications that could lead to smooth performance for handover activity [8]. The OpenWRT [9] assists in the reconfiguration of wireless protocols to enable the SDN programmable control plane in IEEE 802.11 AP to enhance the conventional handover process in a wireless network. Moreover, the emergence of the network function virtualization (NFV) [10] is highly encouraged to implement within the SDN platform to offer multiple benefits to the service operators and the user/mobile terminals (MT). The NFV has been considered as a useful abstraction to hardware functionalities to reduce the infrastructure cost and also helpful to minimize the power consumption. Therefore, it is evident that the SDN and NFV technologies have potential to build the prospective WLAN environment by introducing mobility management features that include seamless handover, wireless resource optimization, centralized management, and fine-grained controllability.

In the current research work, we propose a Logical AP-based Mobility Management (LAPM) scheme for WLAN in the SDN environment with the further functionality of the NFV, which better describe how to design an ideal mobility management environment for WLAN. We construct the logical AP $\left({ }_{\mathrm{L}} \mathrm{AP}\right)$ with an extended SDN/NFV abstraction, which release the IEEE 802.11 protocol stack complexity to forward the operations to the centralized controller. Each ${ }_{\mathrm{L}} \mathrm{AP}$ corresponds to a MT that is associated with the AP. The LAP acts as the virtual AP (VAP), an abstraction of the physical AP ( $\left.{ }_{\mathrm{P}} \mathrm{AP}\right)$, which provides auxiliary network functions. The ${ }_{\mathrm{L}} \mathrm{AP}$ has the ability to maintain same VAP for each associated MT with adjacent pAPs concurrently to enable the seamless handover which can be achieved when MT associated with several ${ }_{\mathrm{p} A P s}$ in the signal overlapping range. We also examine the performance of both conventional handover and our proposed algorithm LAPM. Load balancing among APs that have common signal interference area is also a focus of this research. We build a testing environment, based on the SDN and the NFV technologies, to analyze the functionality of ${ }_{\mathrm{PAP}}$. After implementing the LAPM scheme in a testbed, with the real traffic from users, it is evident from evaluation results that the proposed scheme could significantly minimize the handover latency in WLAN. In addition, LAPM scheme maintains the load balance with the concurrent provision of the seamless handover. This experimental study helps to evaluate the performance of installed ${ }_{\mathrm{p}} \mathrm{APs}$ for further enhancement of the QoE.

The rest of the paper is organized as follows: Section 2 presents the previous studies. Section 3 reports the functionality of mobility management scheme and implementation of LAPM scheme. In Section 4, we present the experimental results. Finally, in Section 5, we conclude the paper.

\section{Related works}

Mobility management is an obligation of the wireless networks. This investigation emphasizes on the WLAN mobility management schemes.

\subsection{The traditional IEEE 802.11 handover schemes}

The IEEE 802.11 AP handoff scheme is defined in three steps [11]: (a) scan the new APs for the association, (b) send the authentication probe to target AP that has strong RSSI, and (c) begin the re-association procedure to connect with the new AP. Consequently, the lengthy traditional handover process is the primary cause of throughput degradation. Besides, there are also certain reasons [12] for packet loss such as electromagnetic interference, transmission impairment, and the hidden terminal problem. In the past decade, various handover approaches have been announced based on the surrounding channels [13] and 
the neighboring graph schemes [14]. These schemes $[13,14]$ perform passive scanning to gather the results. However, channel synchronization during the scanning process declines the frequent handoff in a dense network environment that leads to the poor QoS. Compared with the traditional schemes mentioned above, our proposed scheme collects the neighboring ${ }_{\mathrm{P}} \mathrm{AP}$ information through ${ }_{\mathrm{L}} \mathrm{AP}$ to provide a gateway between the SDN controller and the MT. In our proposed scheme, we restrict the frequent handover of MTs in the closed region APs in order to reduce the latency and the ping-pong ratio.

\subsection{The SDN-based WLAN infrastructure}

Recently, the OpenFlow protocol has been considered in the wireless access network to provide the fine-grained packet control, and SDN makes the separation of underlying physical infrastructure and the network services [15-19]. The OpenRoads [15] was the first project in the SDN-based WLAN environment that enables wireless network slicing using the FlowVisor to assign different SSIDs to the MT. The OpenRoads introduced the OpenFlow-based testbed to control mobility between the WiFi and the WiMax base stations. Mortier et al. [16] proposed a prototype using the OpenFlow protocol to enable flow-based scheme with the fine-grained packet control in home networks. CloudMAC [17] is a distributed architecture that enables transferring the processing of MAC layer functions on the central servers to minimize the IEEE 802.11 AP pressure, and the AP is responsible only for the MAC frames forwarding among the virtual APs using the OpenFlow. Contrarily, CloudMAC did not declare the switching procedure of associated stations among APs simultaneously that is required for per-user handover. Besides, the CloudMAC drives all traffic towards the Cloud which increases the load of the control plane. In contrast, the LAPM scheme provides the seamless mobility without any change on the MTs to reduce the network complexities and the deployment cost. Suresh et al. [18] introduce an SDN framework Odin to empower the seamless mobility in WLAN. The Odin builds the logical virtual access point (LVAP), which is similar to the concept of the virtual APs in the CloudMAC architecture. The LVAP offers a dedicated logical connection to the client with a unique BSSID. During the handoff process, the client does not necessitate re-association with the target AP. The Odin offers seamless handover in a wireless network to reduce the handover delay in comparison with the IEEE 802.11 traditional handover. However, the handover process depends on a formal parameter RSSI which could lead to a load imbalance situation. The SDN-based handover approach [8] proposes for IEEE 802.11WLAN in which the handover procedure depends on neighbor AP response and permits the MT to connect with several APs simultaneously. It also enables fast switching among APs to improve the performance of the video streaming applications. However, the scope of the proposed approach is limited to video streaming applications without the inclusion of video conferencing and peer-to-peer-based applications. SDWLAN [19] presented an architecture that sustains client-unaware handoff on 802.11 AP MAC layer and provides a unified control platform for wireless APs and wired backbone. The wireless access switch (WAS) is a device that configured with the OpenFlow protocol for transferring several module functions of AP onto the centralized controller. However, it is challenging to incorporate WAS into the existing WLAN environment. The proposed LAPM scheme has advantages over the abovementioned SDN-based solutions with the main feature of the mobility management. The LAPM scheme is primarily distinctive from those techniques that support individual mobility of MTs with the load balancing approach among ${ }_{\mathrm{p}} \mathrm{APs}$ in the overlapping signal range without any amendments at the MTs. We performed several experiments on the real testbed to evaluate the performance of handoff and the load balancing in both traditional environment and the SDN-based environment. We will discuss in more detail about the evaluation and results in Section 4 of this research article.

\section{The design and implementation of LAPM}

Our proposed scheme significantly improves the performance of the APs and the MTs without involving any change at the client side. It also offers seamless mobility in which the ${ }_{L} \mathrm{AP}$ can migrate from the source ${ }_{\mathrm{P}} \mathrm{AP}$ to the target ${ }_{\mathrm{PAP}}$ during the handoff session. The traditional RSSI-based association procedure could generate the load imbalance network environment. As depicted in Fig. $1 \mathrm{a}, \mathrm{PAP}^{2}$ associated with the four MTs through individual ${ }_{\mathrm{L}} \mathrm{AP}$ and ${ }_{\mathrm{P}} \mathrm{AP}^{3}$ associated with three MTs, while ${ }_{\mathrm{P}} \mathrm{AP}^{1}$ associated with two mobile terminals. The ${ }_{\mathrm{L}} \mathrm{AP}^{2}$ also exists in the coverage area of ${ }_{\mathrm{PAP}} \mathrm{P}^{1}$, but does not associate because of the less RSSI rather than ${ }_{\mathrm{P}} \mathrm{AP}^{2}$. Contrarily, our proposed approach incorporates workload information of ${ }_{\mathrm{p}} \mathrm{AP}$ during the MT association that ensures a balanced network. As illustrated in Fig. $1 \mathrm{~b},{ }_{\mathrm{L}} \mathrm{AP}^{2}$ can associate with ${ }_{\mathrm{P}} \mathrm{AP}^{1}$ that can reduce the load of ${ }_{\mathrm{p}} \mathrm{AP}^{2}$ and also enhance the throughput of the associated MT.

\subsection{Logical access point abstraction}

The traditional IEEE 802.11 pAP does not permit to track the movement of the associated MTs that disrupted the continuity of established connection, which therefore degrades the service provision. Thus, we construct a logical access point $\left({ }_{\mathrm{L}} \mathrm{AP}\right)$ to abstract the connection, MT associated with AP, as virtual AP (VAP). 


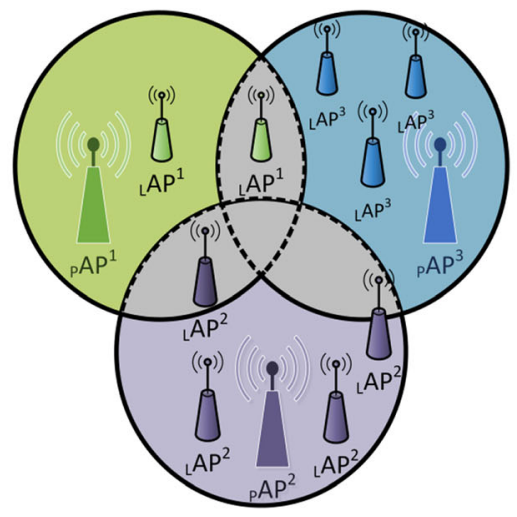

(a) Existing Approach

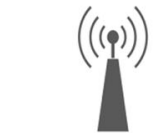

Physical Access Point

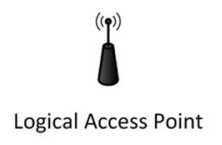

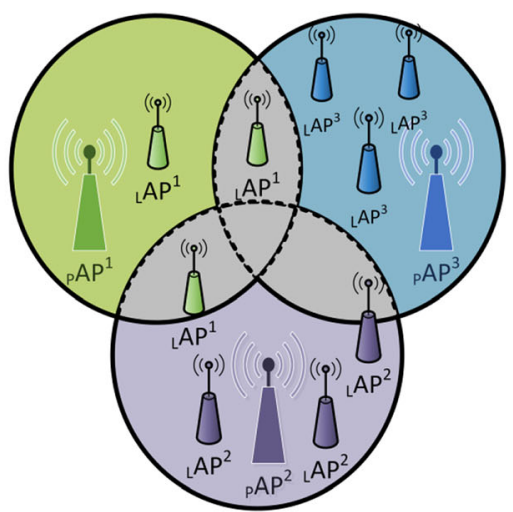

(b) Proposed Approach

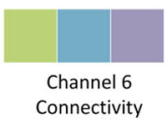

Fig. 1 An illustration of mobility management within the load balancing scenario. a Existing approach. b Proposed approach

The abstraction of physical AP $\left({ }_{\mathrm{P}} \mathrm{AP}\right)$ provides a platform to facilitate the programmable control plane and the mobility management functions. The ${ }_{\mathrm{L}} \mathrm{AP}$ is a logical entity that resides in the ${ }_{\mathrm{P}} \mathrm{AP}$ with an extended SDN/NFV abstraction, which releases the IEEE 802.11 protocol stack complexity to forward the operations to the centralized controller. Furthermore, ${ }_{\mathrm{L}} \mathrm{AP}$ acts as the SDN agent [18] that is responsible for performing the multiple tasks either generated by the SDN controller or the local network. More specifically, the first task is to assign a unique BSSID per client that generates beacon and acknowledgment. Secondly, it sets the initial configuration parameters such as IP address, MAC address, and SSID for the MT and holds the related module information

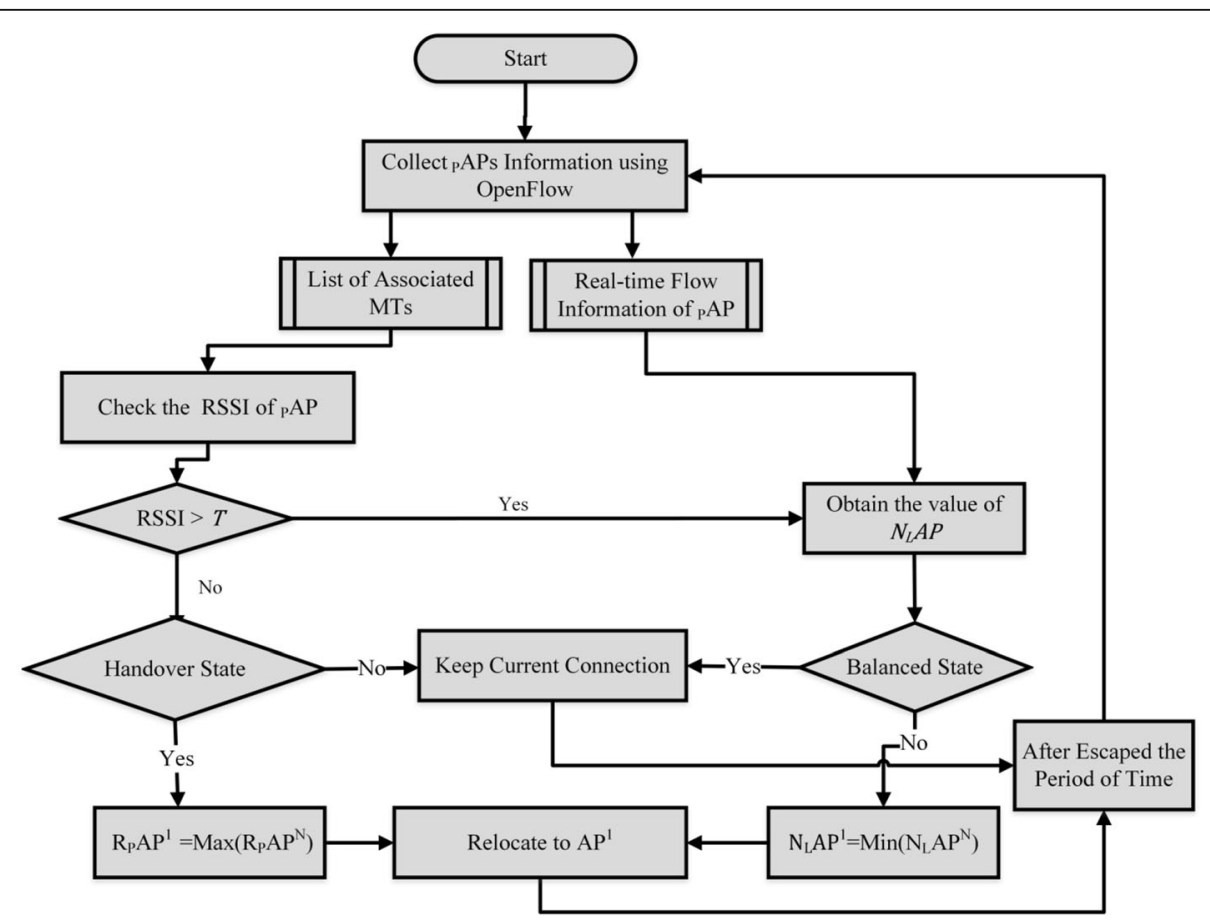

Fig. 2 Flowchart of the LAPM scheme 
Table 1 Specifications and Configuration of pAPS

\begin{tabular}{|c|c|c|c|c|c|c|c|}
\hline & Installed device & Standard & Bandwidth & OS & Protocol & Channel band & Other software tools \\
\hline${ }_{p} \mathrm{AP}^{n}$ & Netgear WNDR3800 & $802.11 \mathrm{n}$ & $40 \mathrm{MHz}$ & OpenWRT rel. 14.07 [9] & OpenFlow Ver. 1.3 & $2.4 \mathrm{GHz}$ & $\begin{array}{l}\text { Open vSwitch Ver. 2.3.90 [23] } \\
\text { Click modular router 2.0.1 [24] }\end{array}$ \\
\hline
\end{tabular}

into the OpenFlow tables. Each ${ }_{\mathrm{P}} \mathrm{AP}$ can host multiple ${ }_{\mathrm{L}}$ APs with different BSSID to give abstraction of a general handover procedure to its associated users. Simultaneously, ${ }_{\mathrm{L}} \mathrm{AP}$ also maintains the same VAP for each associated MT with adjacent ${ }_{\mathrm{PAPs}}$, to enable the seamless handover which can be achieved while MT associated with several ${ }_{\mathrm{P}} \mathrm{APs}$ is in the signal overlapping range. The migration of ${ }_{L} \mathrm{AP}$ among adjacent ${ }_{\mathrm{P}} \mathrm{APs}$ can be performed without a re-association process and also skipped the additional MAC layer procedures that provide an illusion of the consistent connection at the user side. Third, it detects the load of ${ }_{\mathrm{p}} \mathrm{AP}$ using the OpenWRT mwan3 package [9] which includes the outbound traffic load information that we further elaborated in Eq. 1.

$$
N_{\mathrm{L}} \mathrm{AP}=\sum_{n=1}^{N} \mathrm{CS}_{\mathrm{A}}^{n} / \sum_{m=1}^{M} \mathrm{CS}_{\mathrm{P}}^{m}
$$

where $\mathrm{CS}_{\mathrm{A}}$ denotes the current active state of ${ }_{\mathrm{L}} \mathrm{AP}$ that contains the current traffic load (bytes) information of MT within a specified time period. Meanwhile, $\mathrm{CS}_{\mathrm{P}}$ denotes the current passive state of ${ }_{L} \mathrm{AP}$ for the connection interval time and sleeping time. If the current state CS is bigger than a threshold, the ${ }_{\mathrm{L}} \mathrm{AP}$ reflects an active state $\left(C S_{A}\right)$, and if the CS is smaller, the ${ }_{L} A P$ reflects a passive state $\left(C_{\mathrm{P}}\right)$. At the same time, $N$ is the number of ${ }_{\mathrm{L}} \mathrm{AP}$ in $\mathrm{CS}_{\mathrm{A}}$, and $M$ is the number of ${ }_{\mathrm{L}} \mathrm{AP}$ in $\mathrm{CS}_{\mathrm{P}}$.

\subsection{Overall procedure}

The most common method of ${ }_{\mathrm{P}} \mathrm{AP}$ selection is based on RSSI. This approach causes low performance of the network, load imbalance, and also affects the handoff process $[8,19]$. We prefer to collect $N_{\mathrm{L}} \mathrm{AP}$ values described in Eq. 1, and RSSI value that is denoted as $R$. Now, we present the method of threshold $T$ calculation for our proposed scheme. We suppose set $\mathrm{A}=\left\{_{p} \mathrm{AP} \mathrm{P}^{1}\right.$, $\left.{ }_{p} \mathrm{AP}^{2}, \ldots \ldots . . . \mathrm{AP}^{n}\right\}$ that contains $N$ number of installed PAPs in area A. We divided set A into multiple subsets; each subset contains two APs with a common interface area. The reason to put two APs in one subset is to keep the manageability simple in an environment where multiple APs cover a large area. Each subset of APs is installed in sequence to the next subset. Suppose that there are $K$ total number of users in the area $\mathrm{A}$ and $P+Q$ is the number of users in subset area such as $P+Q$ is $\leq K$. We further supposed that users $p$ are associated to ${ }_{p} \mathrm{AP}^{j}$ and $Q$ users are associated to ${ }_{p} \mathrm{AP}^{k}$ in the subarea $\beta$.

$$
\begin{aligned}
& \alpha=\left\{{ }_{p} \mathrm{AP}^{i},{ }_{p} \mathrm{AP}^{j}\right\} ; \beta=\left\{{ }_{p} \mathrm{AP}^{j},{ }_{p} \mathrm{AP}^{k}\right\} ; \delta=\left\{{ }_{p} \mathrm{AP}^{k},{ }_{p} \mathrm{AP}^{l}\right\} \forall \alpha, \beta, \delta \in A \\
& \text { where } \quad\{i, j, k, l\}=1,2,3 \cdots(P+Q) \quad, \quad i \neq j \neq k \neq l
\end{aligned}
$$

We suppose $n, m$ for the number of mobile terminals. For each user $k$, we use a variable $M T_{k j}$ to denote its association with $\mathrm{AP}^{j}$. Formerly supposing the mobile terminal set $S_{p} \mathrm{AP}^{j}=\left\{M T_{1 j}, M T_{2 j} \ldots M T_{n j}\right\}$ are connected $\mathrm{AP}^{j}$. Similarly, the set $S_{p} \mathrm{AP}^{i}=\left\{M T_{1 i}, M T_{2 i} . . M T_{m i}\right\}$ are connected $\mathrm{AP}^{i}$. $\left\{\left\{M T_{1 j}, M T_{2 j} \ldots M T_{n j}\right\},\left\{M T_{1 i}, M T_{2 i} \ldots\right.\right.$ $\left.\left.M T_{m i}\right\}\right\}$ are connected A.

$$
T=\frac{1}{M+N}\left(\sum_{n=1}^{N} R_{M T_{n j}}+\sum_{m=1}^{M} R_{M T_{m i}}\right)
$$

Figure 2 presents the flowchart of the proposed LAPM scheme. In the initialization phase, the controller gets information from the agent about MT connected to ${ }_{\mathrm{p}} \mathrm{APs}$ that belongs to A. Afterwards, it performs scanning

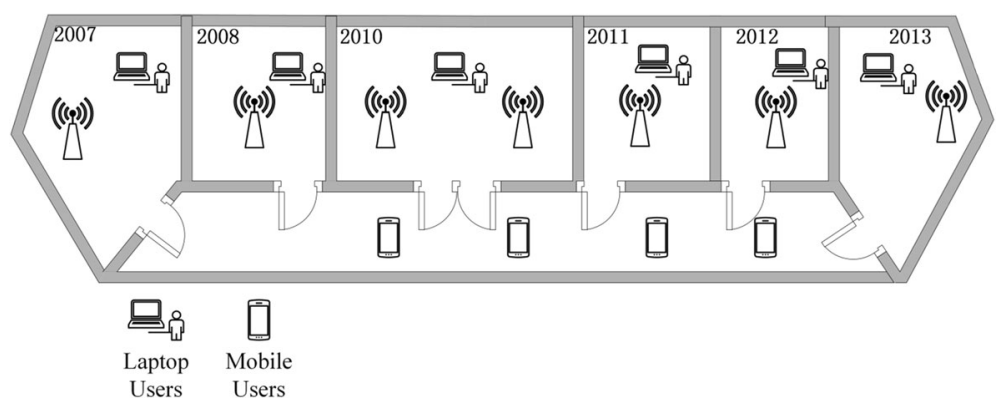

Fig. 3 Sketch of the WLAN testbed deployed at the Xinke building, CQUPT campus 
(a)

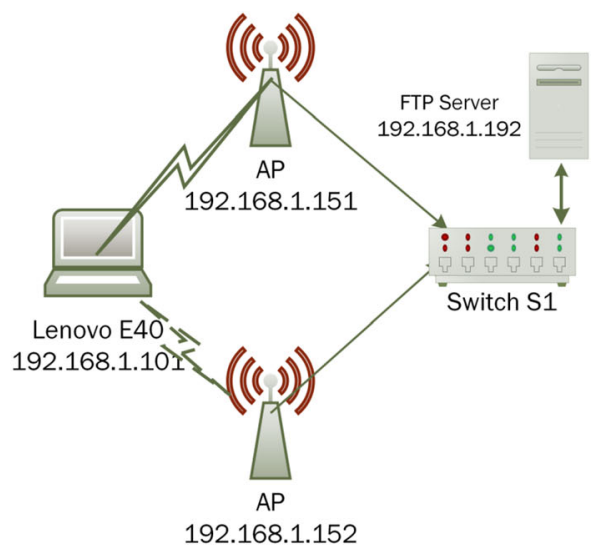

(b)

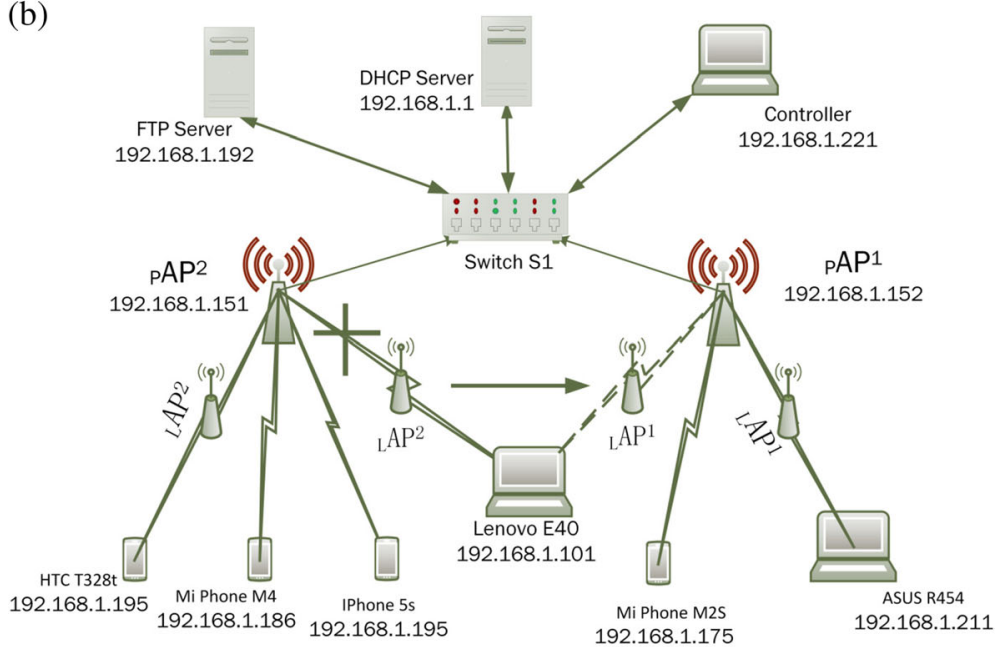

Fig. 4 Real testbed scenario of mobility management in WLAN. a Traditional handover scheme. b LAPM handover scheme implementation

(a)

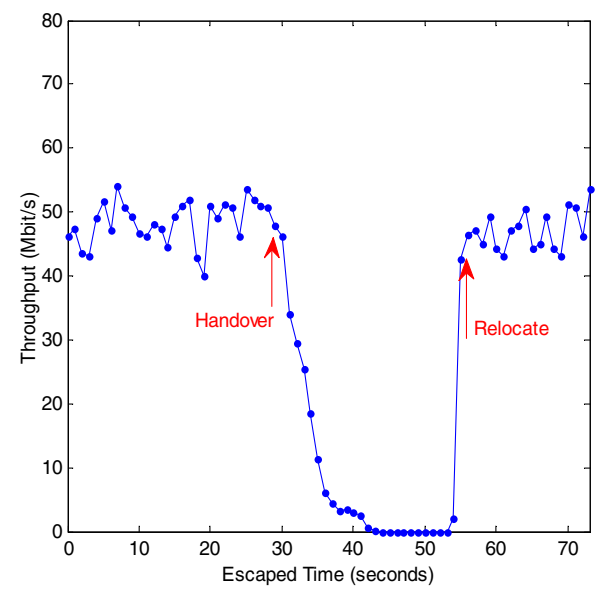

(b)

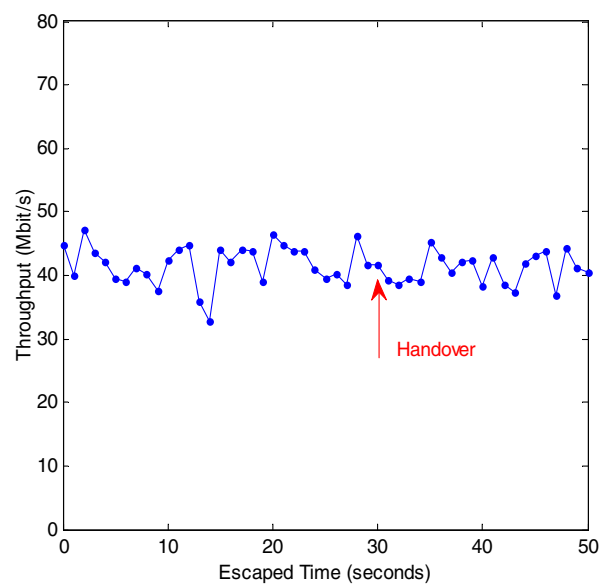

Fig. 5 Performance analysis of handover cost. a Traditional handover. b LAPM-based handover 

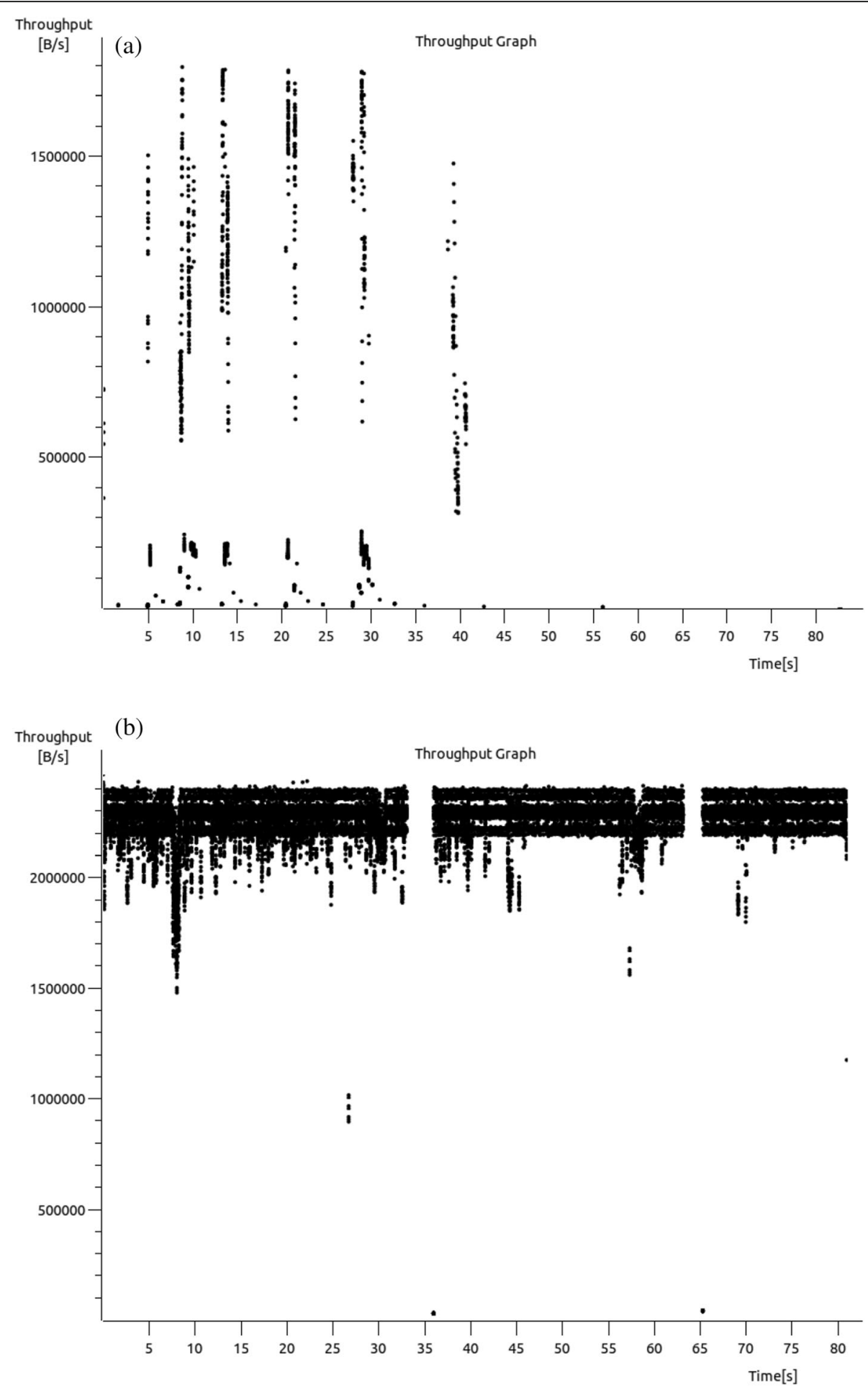

Fig. 6 Instantaneous TCP stream at the receiver's side. a Traditional IEEE 802.11. b LAPM

followed by a comparison between the received values using pre-defined parameters of the algorithm. If the current RSSI of MT is greater than the threshold $T$ (Eq. 3), then it compares the $N_{\mathrm{L}} \mathrm{AP}$ with the ${ }_{\mathrm{P}} \mathrm{AP}$, and if comparison generates a false value, then it enters into the mobility phase. Moreover, MT further scans ${ }_{\mathrm{p}} \mathrm{AP}$ with improving RSSI and $N_{\mathrm{L}} \mathrm{AP}$. As illustrated in Fig. 1a, MT within the signal interference zone can select the best AP in the area. However, if the user performs handoff for more than three times, it demonstrates that the 
user fails to find a better AP. Therefore, we set the handover state to FALSE and exit from the comparison process. Furthermore, if the handoff state is TRUE, it will continue to the next steps.

\subsection{Implementation of LAPM scheme}

The LAPM scheme has implemented in real WLAN at Xinke building in Chongqing University of Posts and Telecommunication (CQUPT) campus. The developed testbed consists of ${ }_{p}$ APs in each office room, and all adjacent ${ }_{p} \mathrm{APs}$ have a signal overlapped area to provide continuous mobility. For the initial experiments, we randomly select one ${ }_{\mathrm{P}} \mathrm{AP}$ from room 2010 and another from room 2011; the detailed specification of selected ${ }_{p}$ APs is given in the Table 1.

We deployed a LAPM controller on the server (dual four-core CPU, 16 GB RAM) to run the Ubuntukylin14.04, and the Floodlight version 1.2 [20]. The Floodlight is an open source controller that provides several functionalities such as it manages the network, monitors the packet flow control, enables the modification in packet headers, and forwards the incoming packets on specific port(s) as per defined rules. Furthermore, we develop some modules in the Floodlight controller to perform multiple tasks such as obtaining the initial information of ${ }_{\mathrm{P}} \mathrm{AP}$ including RSSI and CS information by executing algorithm1, and the LAPM controller also performs processing to enable additional MAC layer functions. Subsequently, algorithm2 is responsible to perform the mobility tasks related to ${ }_{\mathrm{PAP}}$ such as disable ${ }_{\mathrm{L}} \mathrm{AP}$ or association of ${ }_{L} A P$, mapping of ${ }_{L} A P$ to adjacent ${ }_{P} A P$, and migration of ${ }_{L} A P$ between ${ }_{P} A P$ if load imbalanced state occurs.

\section{Evaluation cases and experimental results}

We have evaluated the performance of the LAPM scheme in real WLAN testbed at the technology building in CQUPT campus by monitoring the real traffic as illustrated in Fig. 3. Ping and iPerf [21] measure the traffic load of AP and MT throughput. However, the packet transfer rate of cell phones and laptops could differ due to hardware capacity. We developed two scenarios to perform the experiments. In the first scenario, we compare the performance of LAPM handover with traditional WLAN handover while accessing the same file server as illustrated in Fig. 4. We configured Xlight FTP server and selected the large file more than $1 \mathrm{~GB}$ to make a certain downloading process that should continue until handoff completion. The second scenario is about an experiment that was carried out on individual pAPs to measure the traffic load. In this case, the proposed scheme perceived load imbalanced and repositioned the MT from overloaded AP to adjacent lessloaded AP. The results showed the balanced traffic load on ${ }_{\mathrm{P}} \mathrm{APs}$ and improvement of throughput on MT and also minimized the packet loss ratio.

\subsection{Case 1: handover between ${ }_{\mathrm{PAPs}}$}

In this experiment, we compared the performance of fixed RSSI-based handover algorithm with proposed LAPM algorithm. In the first test, we evaluate ${ }_{\mathrm{P}} \mathrm{AP}$ handover performance in the traditional environment as shown in Fig. 4a. The process of connecting to ${ }_{\mathrm{P}} \mathrm{AP}$ is a four-way handshake according to IEEE $802.11 \mathrm{n}$ standard. During the experiment, the user has assigned a dynamic IP on its first connection with the network. The handoff procedure does not require changing the assigned address, which therefore saves the time of users for

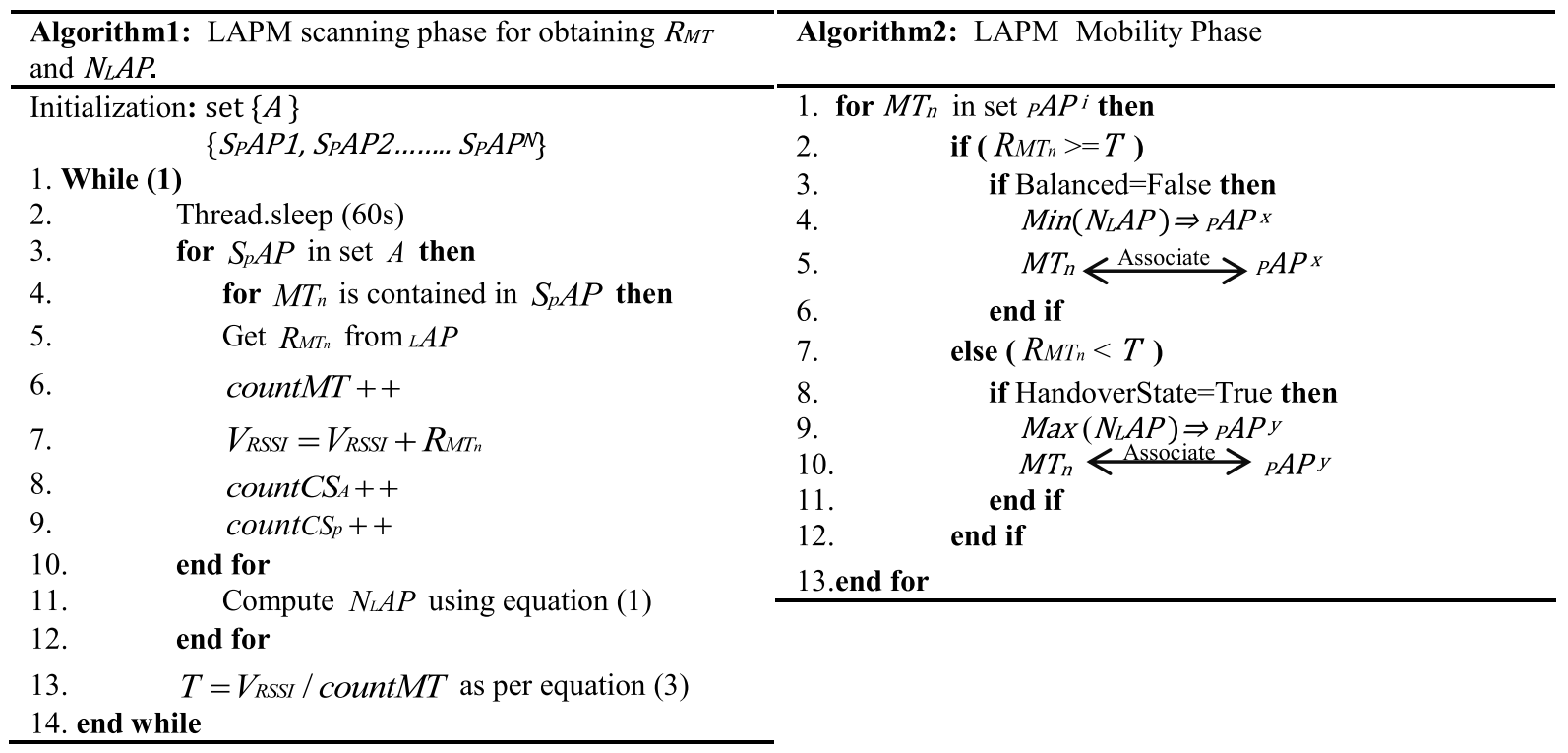


inquiring IP addresses. Initially, Lenovo E40 established a connection with ${ }_{\mathrm{p}} \mathrm{AP}$ (192.168.1.151) and activates the process of data downloading from the FTP server (192.168.1.192) at time $t=0 \mathrm{~s}$ with an average throughput rate of approximately $45 \mathrm{Mbits} / \mathrm{s}$. Meanwhile, the user has performed a handover to ${ }_{\mathrm{P}} \mathrm{AP}$ (192.168.1.152) after an elapsed time of $30 \mathrm{~s}$; Fig. 5a indicates the throughput regarding the elapsed time during the fixed RSSI handover process. The imitation of the handover process and connection interruption during the downloading process at $t=30 \mathrm{~s}$ significantly decrease the throughput from 46.3 to 3.28 Mbits/s in $8 \mathrm{~s}$. Moreover, the throughput reached at zero level after elapsed time $t=43 \mathrm{~s}$, because the connection between MT and network was lost for an extended time. The recovery time is about $10 \mathrm{~s}$ in the traditional WLAN environment caused by a IEEE 802.11 standard handover mechanism that includes detection, probing, and re-association processes (Fig. 5a).

The second experiment was performed in the SDNbased testbed as illustrated in Fig. 4b. We selected two ${ }_{\mathrm{p}} \mathrm{APs}$, the detail specification is reported in Table 1, connected with the OpenFlow switch S1 underneath the SDN controller (192.168.1.221). Initially, Lenovo E40 established a connection with ${ }_{\mathrm{P}} \mathrm{AP}^{2}$ through ${ }_{\mathrm{L}} \mathrm{AP}^{2}$ at $t=$ $0 \mathrm{~s}$ and initiated the file transferring process with an average throughput of approximately $40 \mathrm{Mbits} / \mathrm{s}$. As the handover initiated at $t=30 \mathrm{~s}$, the ${ }_{\mathrm{L}} \mathrm{AP}^{2}$ migrates the credentials to target ${ }_{\mathrm{P}} \mathrm{AP}^{1}$ for newly associated ${ }_{\mathrm{L}} \mathrm{AP}^{1}$, and ${ }_{L}$ AP handoff almost did not produce any delay in packet transmission that sustained the constant throughput average at $40 \mathrm{Mbits} / \mathrm{s}$ as shown in Fig. 5b. In comparison with the fixed RSSI-based handover, our proposed handover scheme produces a stable and nearly zero latency transition among the ${ }_{\mathrm{p}} \mathrm{APs}$ while retaining the network

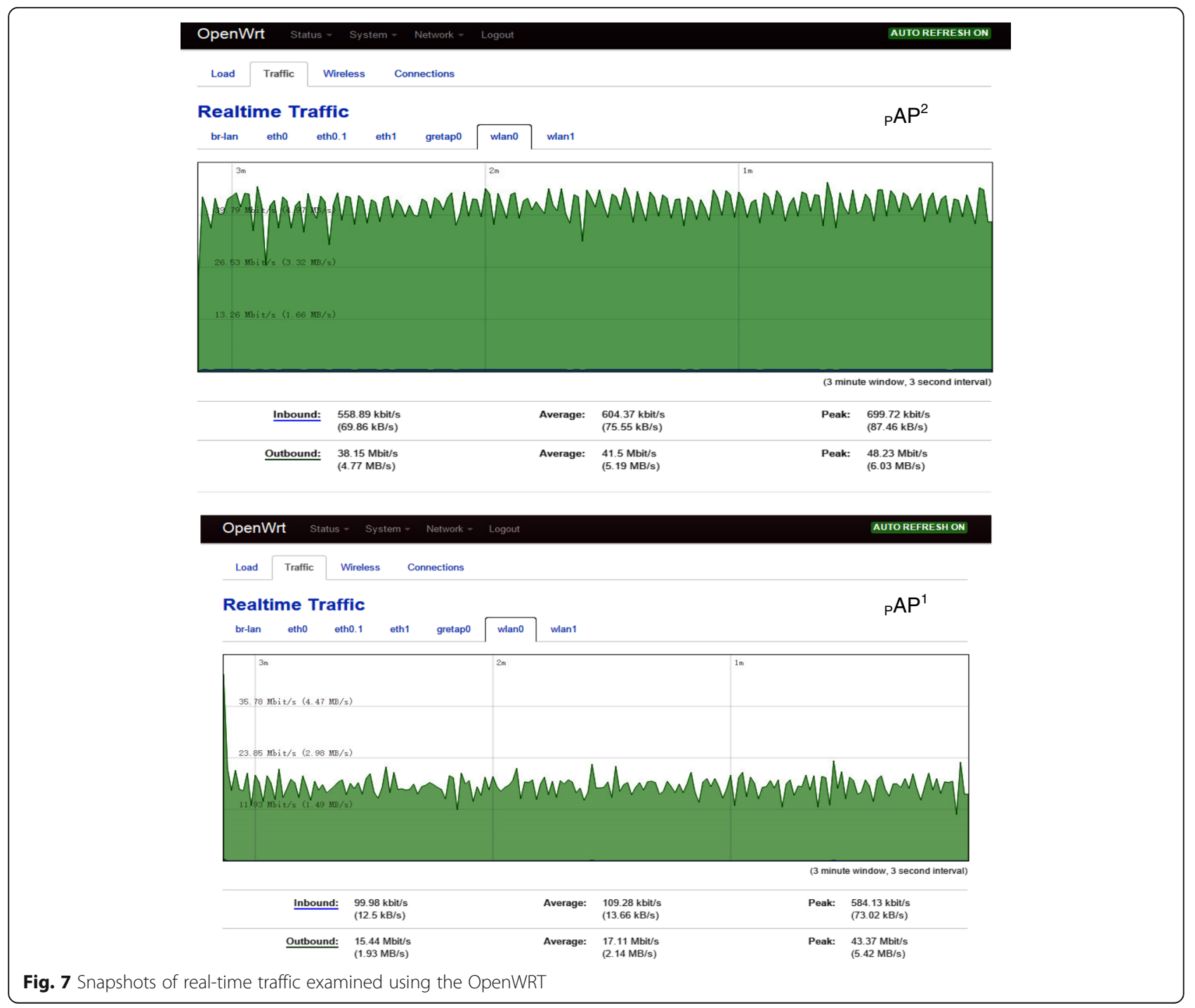


throughput at a minimum. Besides, Fig. 6 demonstrates the TCP throughput consumption between MTs and server measured by iPerf [21] for $100 \mathrm{~s}$ that is captured by Wireshark [22]. In Fig. 6a, the server's receiving traffic flow throughput is degraded because whenever MT performed the traditional handover process, it could face a packet drop problem as illustrated in Fig. 5a. On the other hand, the LAPM scheme (Fig. 6b) successfully maintained the throughput between MTs and the server. Therefore, the throughput is graded and higher than the traditional scheme. It is also worth noting that load imbalanced network also degrades the throughput that will be discussed in Section 4.2.

\subsection{Case 2: LAPM scheme for MT relocation and load balancing}

The goal of the next experiment is to demonstrate how the LAPM scheme can enhance the network throughput of those MTs that were overlapping in region A. We installed two ${ }_{\mathrm{P}} \mathrm{APs}$ with the same overlapping region and selected six MTs for this experiment.

Initially, four mobile terminals were connected with the ${ }_{\mathrm{p}} \mathrm{AP}^{2}$ because they received strong RSSI from ${ }_{\mathrm{p}} \mathrm{AP}^{2}$, and the remaining two MTs were connected with ${ }_{\mathrm{P}} \mathrm{AP}^{1}$ as described in Fig. 1a. We performed a test on real-time traffic and plot the results at a 3-min data rate through OpenWRT as demonstrated in Fig. 7. The inbound traffic of ${ }_{\mathrm{p}} \mathrm{AP}^{2}$ was $69.86 \mathrm{kB} / \mathrm{s}$ while ${ }_{\mathrm{p}} \mathrm{AP}^{1}$ was $12.5 \mathrm{kB} / \mathrm{s}$. The average traffic load of ${ }_{\mathrm{P}} \mathrm{AP}^{2}$ was $41.5 \mathrm{Mb} / \mathrm{s}$ that was closest to the peak value. Conversely, the bandwidth usage on neighbor ${ }_{\mathrm{P}} \mathrm{AP}^{1}$ was $17.11 \mathrm{Mbits} / \mathrm{s}$ which was far less than that on ${ }_{\mathrm{p}} \mathrm{AP}^{2}$. This imbalanced situation of ${ }_{\mathrm{p}} \mathrm{APs}$ is reported in Fig. 7. The LAPM scheme detected the load imbalanced state and selects one MT (Lenovo E40 as ${ }_{\mathrm{L}} \mathrm{AP}^{2}$ ) which is located in the overlapping region as shown in Fig. 1a. Lenovo E40 established a connection with the FTP server at $t=0$ through ${ }_{\mathrm{p}} \mathrm{AP}^{2}$. Using iPerf [21], we got the average throughput rate of $10 \mathrm{Mbits} / \mathrm{s}$ before $t=29$ as shown in Fig. 8a. At time $t=30 \mathrm{~s}$, handover was triggered
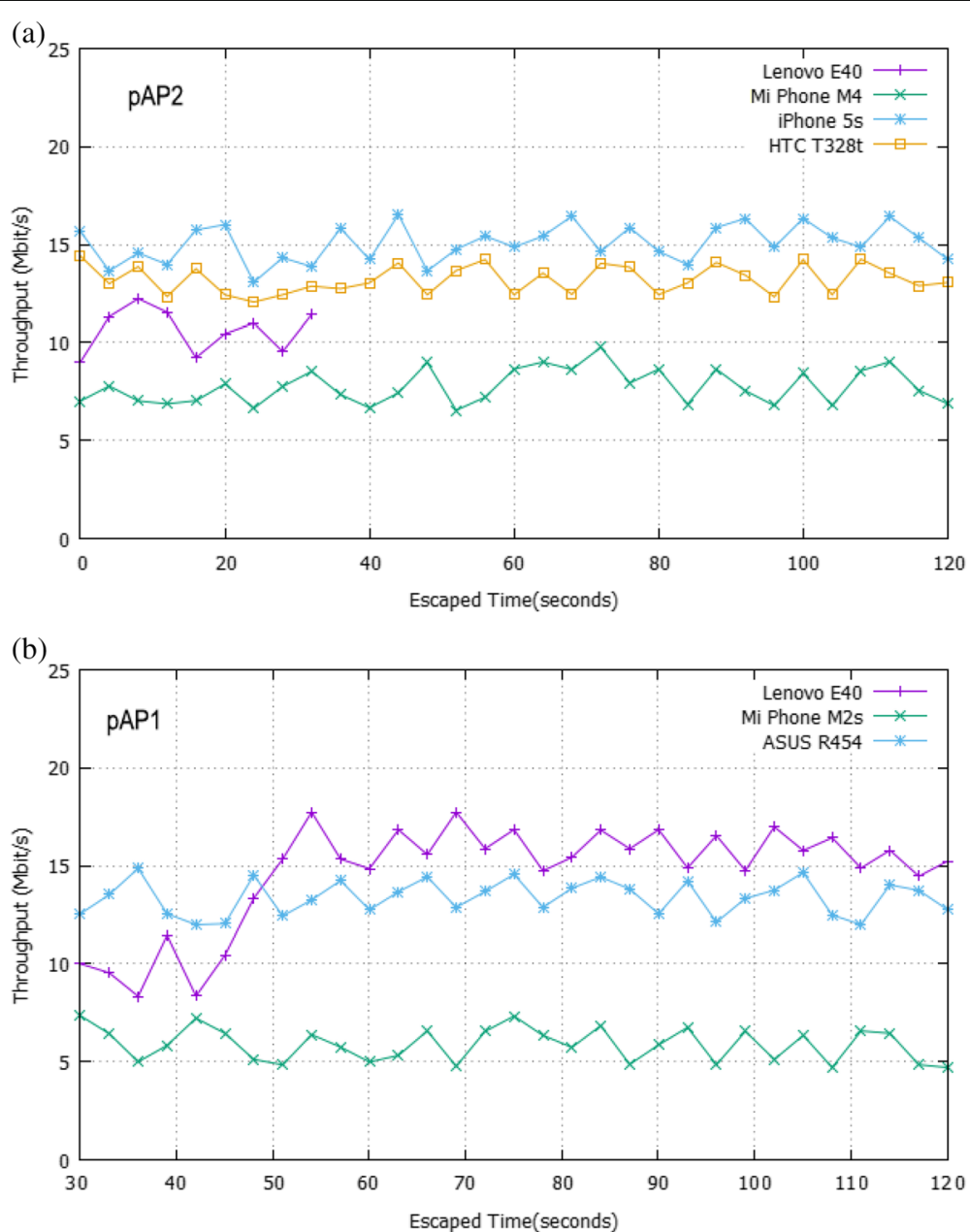

Fig. 8 Throughput analysis during the relocation process of the MT from one ${ }_{p} A P$ to adjacent ${ }_{p} A P$ invoked by the LAPM scheme. a Before handover. b After handover 
by the LAPM scheme without the awareness of the user, and relocated ${ }_{\mathrm{L}} \mathrm{AP}^{2}$ from ${ }_{\mathrm{P}} \mathrm{AP}^{2}$ to ${ }_{\mathrm{P}} \mathrm{AP}^{1}$. After the repositioning process of Lenovo E40, its throughput grew quickly, and the average throughput was approximately $15 \mathrm{Mbits} / \mathrm{s}$ as depicted in Fig. 8b. In comparison with the fixed RSSI scheme, the LAPM scheme also improves throughput by $5 \mathrm{Mbits} / \mathrm{s}$ (Fig. 8b) at Lenovo E40. The results validate that our proposed scheme improves the throughput of the MT and simultaneously enhances the overall performance of the connected MTs.

In the LAPM scheme, the load balancing among $\mathrm{PAPs}$ results in a decrease of the outbound traffic about 12.68 Mbits $/ \mathrm{s}$ at ${ }_{\mathrm{p}} \mathrm{AP}^{2}$, the average data rate at ${ }_{\mathrm{p}} \mathrm{APs}$ was about $28.82 \mathrm{Mb} / \mathrm{s}$ as illustrated in Fig. 9. Meanwhile, the average inbound traffic rate of ${ }_{\mathrm{p}} \mathrm{AP}^{2}$ reduced from 75.55 to $26.96 \mathrm{kB} / \mathrm{s}$ that was good enough for other connected MTs. Simultaneously, the average outbound traffic load of ${ }_{\mathrm{PAP}}{ }^{1}$ was significantly expanded from 17.11 to $25.36 \mathrm{Mbits} / \mathrm{s}$ as reported in Fig. 9. Hence, the network infrastructure was balanced and feasible to provide optimal services to its existing and new users.

LAPM manifests significant advantages compared to the WiFi legacy scheme regarding the throughput and load balancing. In order to further evaluate the precise load balancing performance, we analyzed the packet loss percentage in both traditional WLAN and LAPM-based WLAN. In the first test, we did not apply a load balancing approach and selected six MTs as test devices. The selected MTs are laptop users, and their fixed positions were fixed in room 2008 (Fig. 3). As shown in Fig. 3, single ${ }_{\mathrm{P}} \mathrm{AP}$ was deployed in room 2008, so all MTs received strong RSSI and associated with that ${ }_{\mathrm{p}} \mathrm{AP}$ sequentially. In traditional WLAN, we observed the packet loss ratio for individual MT. In the event of two MT associations, the packet loss was below
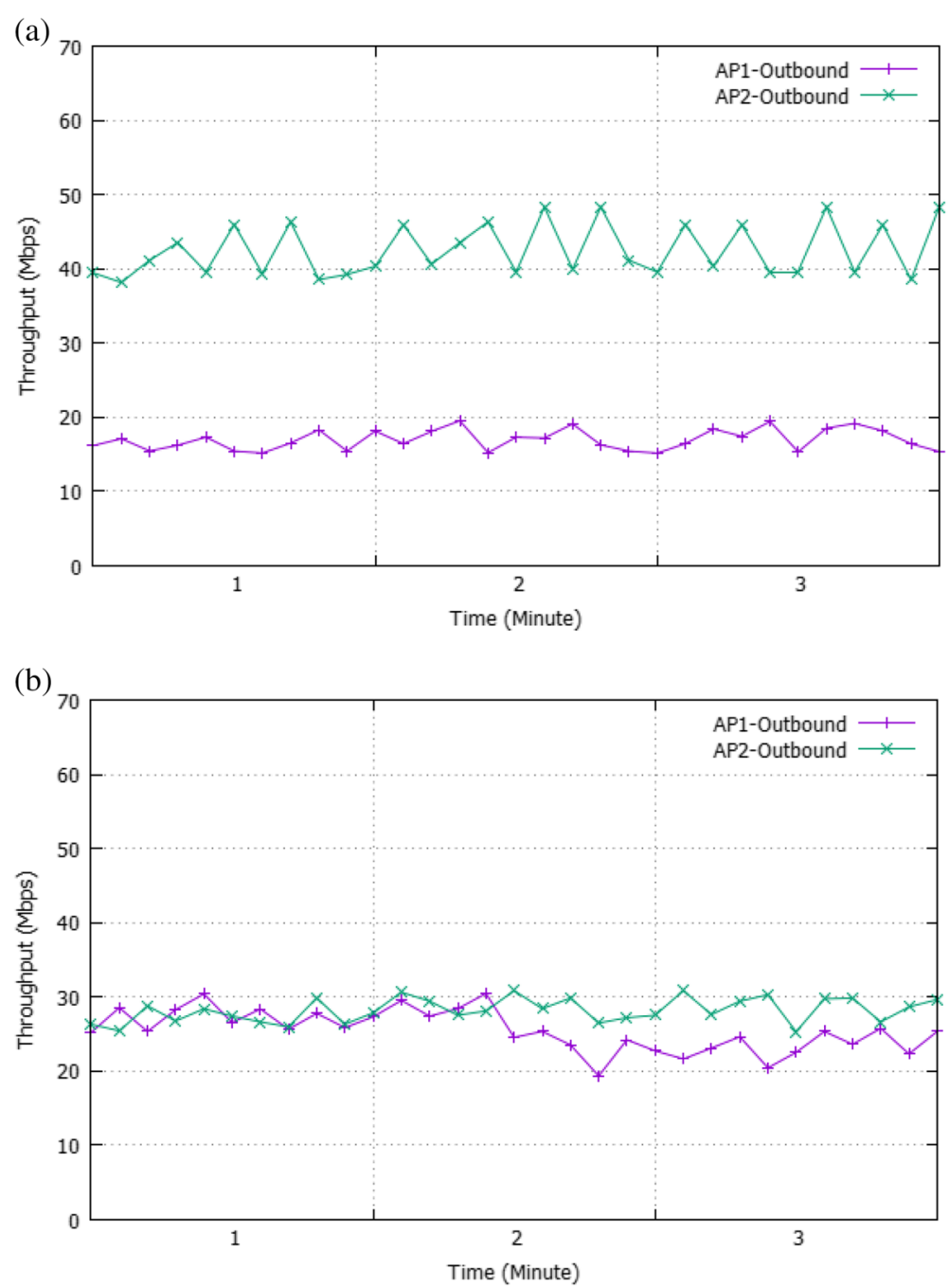

Fig. 9 Demonstration of the real-time traffic analysis of pAPs after the implementation of the LAPM scheme. a Load imbalanced situation among adjacent $\mathrm{p} A P s$. $\mathbf{b}$ Load balanced situation among adjacent $\mathrm{p} A P s$ 
$10 \%$, and the increasing rate of packet loss as the number of associated stations increased is quite notable that is above $60 \%$ (Fig. 10a). There is a strong relationship between the pAP workload (outbound traffic) and the packet loss ratio. Consequently, it also affects the throughput of the MTs as depicted in Fig. 8. In the LAPM scheme, we adopted and repeated the association procedure in the first setup. Three MTs associated with deployed ${ }_{\mathrm{P}} \mathrm{AP}$ in room 2008 and remaining three MTs associated with adjacent ${ }_{\mathrm{PAP}}$ that was deployed in room 2010. Thus, LAPM has managed the overloading of the adjacent ${ }_{\mathrm{p}} \mathrm{APs}$ same as we exposed in Fig. 1b, which remarkably reduce the overall packet loss percentage (Fig. 10b) in comparison with the traditional WLAN. The packet loss is significantly decreased and controllable, and the ratio did not exceed $20 \%$ which manifests that LAPM minimized the packet loss nearly $40 \%$ in comparison with WLAN.

Figure 11 reports the TCP and UDP throughput comparison between the Odin and the LAPM schemes. We analyzed both schemes based on the TCP uploading and downloading process. In Odin-based WLAN, the observed average TCP downloading rate was approximately $1.17 \mathrm{Mbps}$ and average TCP uploading rate was approximately $5.40 \mathrm{Mbps}$ (Fig. 11a). In LAPM-based WLAN, the observed average TCP downloading throughput was $2.19 \mathrm{Mbps}$ and average TCP uploading throughput was approximately $9.92 \mathrm{Mbps}$ (Fig. 11b). We performed the same experiment with the UDP, which demonstrates that the Odin UDP average uplink was $9.37 \mathrm{Mbps}$ and average downloading throughput was 10.69 Mbps (Fig. 11b). Contrarily, in the LAPM-based WLAN experiment, the observed average UDP uplink was 12.34 Mbps and average downloading throughput was $13.51 \mathrm{Mbps}$ (Fig. 11b). The observed statistics of the experiment shows that the throughput of LAPM-based WLAN is significantly higher than that of the Odin-based WLAN (Fig. 11). The evaluation results reveal that our LAPM-based WLAN scheme efficiently increases the throughput of the network and significantly decreases the packet loss

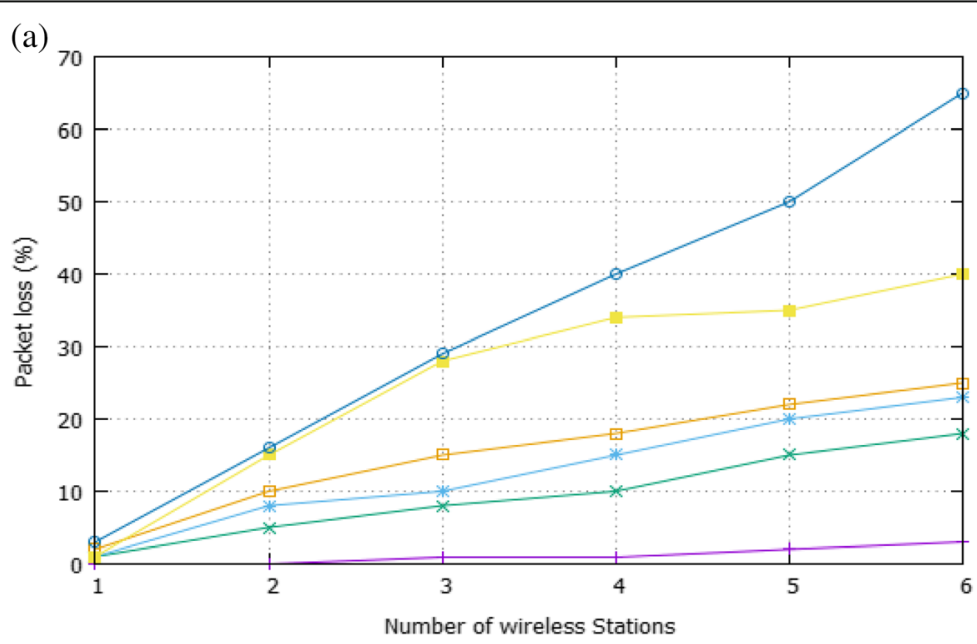

(b)

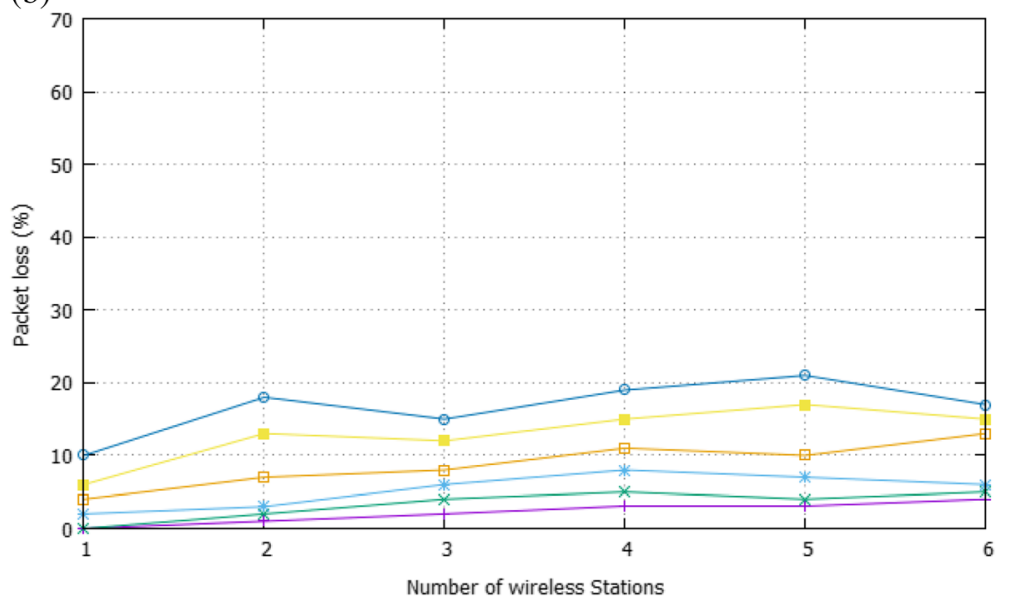

Fig. 10 Comparison of packet loss ratio between traditional WLAN and LAPM scheme-based WLAN. a Traditional WLAN. b LAPM scheme-based WLAN 

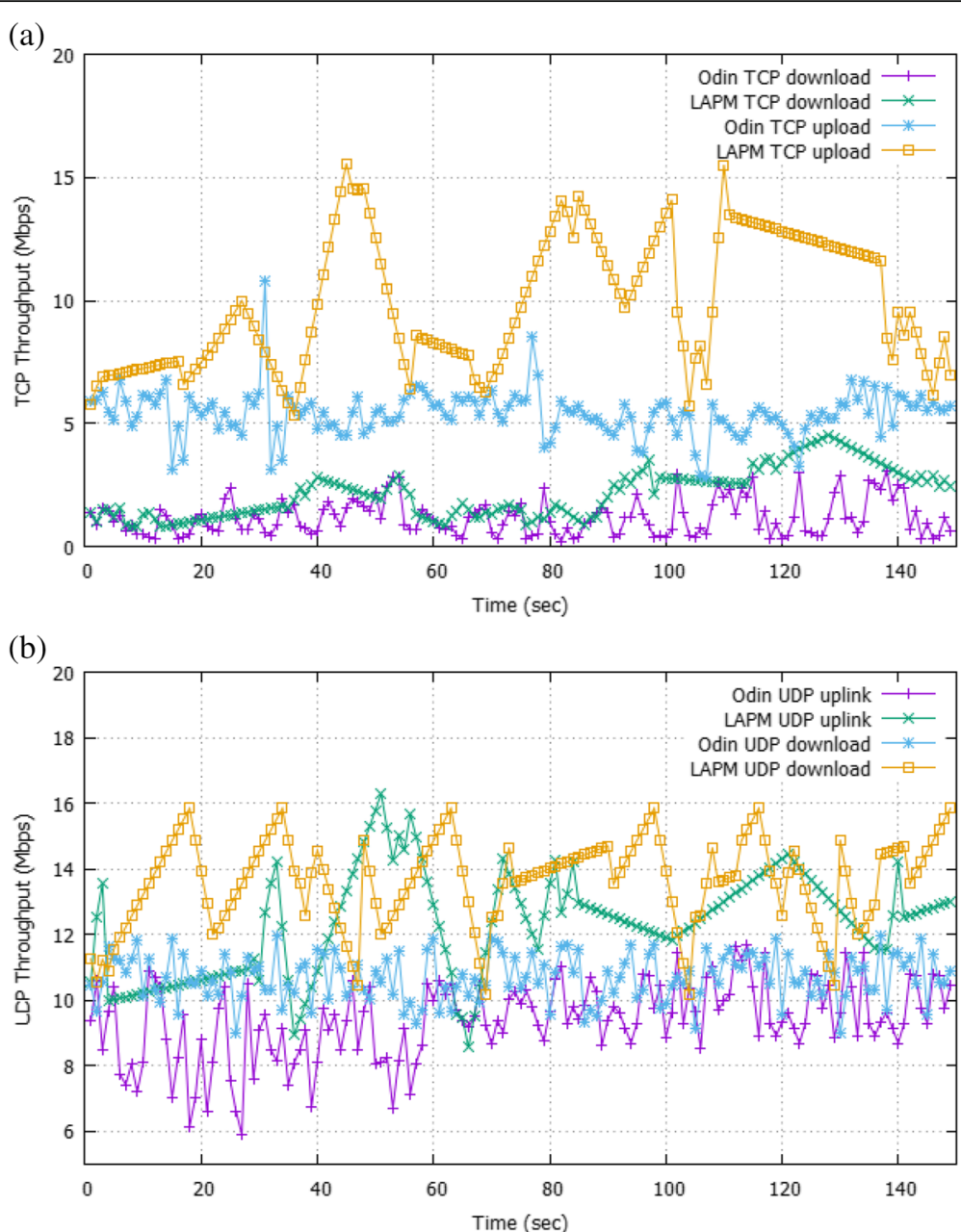

Fig. 11 Throughput comparison between the Odin and the LAPM scheme based on TCP and UDP throughput. a TCP throughput analysis. b UDP throughput analysis

ratio while simultaneously balances the network by fair distribution of the resources.

\section{Conclusions}

The emergence of the SDN/NFV paradigm provides us with a new chain of innovative prospects for solving the mobility issues in the traditional WLAN. To address the problems of seamless handover and MT association with overloaded APs in the signal-overlapping area, in this paper, we proposed an integrated mobility management scheme which considers both seamless handover and load balancing. Furthermore, the proposed scheme can be easily deployed without involving any modification at the client side. The proposed scheme makes it feasible for the users to connect with the best available AP. Our experiments demonstrate how to create ${ }_{\mathrm{L}} \mathrm{AP}$, assign ${ }_{\mathrm{L}} \mathrm{AP}$, and make the migration of ${ }_{\mathrm{L}} \mathrm{AP}$ according to a real-time decision algorithm that was executed at the SDN controller. The evaluation results reveal that our LAPMbased WLAN scheme efficiently increases the throughput of the network and significantly decreases the packet loss ratio while simultaneously balances the network. For further study, the mobility management scheme will be investigated for a power saving module, and location awareness would be integrated into the current scheme, LAPM, to offer more true mobility in the future Internet architecture.

\section{Acknowledgements}

This work was supported by National Nature Science Foundation (61402065,61501075), Prospective Research Project on Future Networks (BY2013095-2-03) fund by Jiangsu Future Networks Innovation Institute and Project Foundation of Chongqing Municipal Education Committee (No. KJ1500429).

\section{Authors' contributions}

SMMG (PhD student) is the main author of current paper, he has raised the main idea, proposed the scheme, and wrote the manuscript. TH is the PhD advisor, she has suggested improvements in the main idea of the research and writing of manuscript. WJ has designed the experiments for seamless handover, and $\mathrm{HMH}$ has assisted to execute the experiments and gathered the results for evaluation. ZG has analyzed the proposed the approach and suggested improvements in the implementation and reviewed the content of the paper. CX has contributed with the expert's advice for developing real testbed in the WLAN. All authors read and approved the final manuscript. 


\section{Competing interests}

The authors declare that they have no competing interests.

\section{Publisher's Note}

Springer Nature remains neutral with regard to jurisdictional claims in published maps and institutional affiliations.

Received: 19 November 2016 Accepted: 1 April 2017

Published online: 13 April 2017

\section{References}

1. Juniper Research Report (2015), http://www.mobileeurope.co.uk/Press-Wire/ mobile-data-traffic-to-hit-197-000-petabytes-by-2019-mainly-offloaded-to-wifi. Accessed 14 Sept 2016

2. IF Akyildiz, DM Gutierrez-Estevez, EC Reyes, The evolution to $4 \mathrm{G}$ cellular systems: LTE-Advanced. Phys Commun 3, 217 (2010)

3. S Sezer, S Scott-Hayward, P-K Chouhan, B Fraser, D Lake, J Finnegan, N Viljoen, M Miller, N Rao, Are we ready for SDN? Implementation challenges for software-defined networks. Commun Mag IEEE 51, 36 (2013)

4. IEEE Computer Society, LAN/MAN Standards Committee, IEEE Standard for Information Technology Part 11, Wireless LAN Medium Access Control (MAC) and Physical Layer (PHY) Specifications Part 11, 2007

5. MA Ergin, K Ramachandran, M Gruteser, Understanding the effect of access point density on wireless LAN performance. in Proc. 13th Annu. ACM Int. Conf. Mob. Comput. Netw (ACM, 2007), pp. 350-353.

6. Open Networking Foundation. https://www.opennetworking.org/. Accessed 18 Nov 2016

7. H Farhady, H Lee, A Nakao, Software-defined networking: a survey. Comput Netw 81, 79 (2015)

8. P Dely, A Kassler, L Chow, N Bambos, N Bayer, H Einsiedler, C Peylo, D Mellado, M Sanchez, A software-defined networking approach for handover management with real-time video in WLANs. J Mod Transp 21, 58 (2013)

9. OpenWrt-mwan3 package (2015), https://wiki.openwrt.org/doc/howto/ mwan3\#capabilities. Accessed 10 Nov 2016

10. C Cui, H Deng, D Telekom, U Michel, H Damker, Network functions virtualization, an introduction, benefits, enablers, challenges \& call for action. White Pap. (2015)

11. A Mishra, M Shin, W Arbaugh, An empirical analysis of the IEEE 802.11 MAC layer handoff process. ACM SIGCOMM Comput Commun Rev 33, 93 (2003)

12. DJ Leith, D Malone, Field measurements of 802.11 collision, noise and hidden-node loss rates, in Model. Optim. Mob. Ad Hoc Wirel. Netw. WiOpt 2010 Proc. 8th Int. Symp. On (IEEE, 2010), pp. 412-417.

13. I Ramani, S Savage, SyncScan: practical fast handoff for 802.11 infrastructure networks, in INFOCOM 2005 24th Annu. Jt. Conf. IEEE Comput. Commun. Soc. Proc. IEEE, 2005, pp. 675-684

14. S-H Park, H-S Kim, C-S Park, J-W Kim, S-J Ko, Selective channel scanning for 1042 fast handoff in wireless LAN using neighbor graph, in IFIP Int. Conf. Pers. Wirel. Commun. (Springer, 2004), pp. 194-203.

15. K-K Yap, M Kobayashi, R Sherwood, T-Y Huang, M Chan, N Handigol, N McKeown, OpenRoads: empowering research in mobile networks. ACM SIGCOMM Comput Commun Rev 40, 125 (2010)

16. R Mortier, T Rodden, T Lodge, D McAuley, C Rotsos, AW Moore, A Koliousis, I Sventek, Control and understanding: owning your home network, in Commun. Syst. Netw. COMSNETS 2012 Fourth Int. Conf. On (IEEE, 2012), pp. 1-10.

17. P Dely, J Vestin, A Kassler, N Bayer, H Einsiedler, C Peylo, Cloudmac-an Openflow based architecture for 802.11 MAC layer processing in the cloud, in Globecom Workshop GC (IEEE, 2012), pp. 186-191.

18. L Suresh, J Schulz-Zander, R Merz, A Feldmann, T Vazao, Towards programmable enterprise WLANS with Odin, in HotSDN'12 Hels. Finl. Copyr ACM, 2012, pp. 115-120

19. D Zhao, M Zhu, M Xu, SDWLAN: a flexible architecture of enterprise WLAN for client-unaware fast AP handoff, in Comput. Commun. Netw. Technol. ICCCNT 2014 Int. Conf. On, 2014, pp. 1-6

20. OpenFlow Hub and Project Floodlight Documentation. https://floodlight. atlassian.net/wiki/. Accessed 18 Oct 2016

21. iPerf - The TCP, UDP and SCTP network bandwidth measurement tool. https://iperf.fr/. Accessed 18 Oct 2016

22. Wireshark Go Deep. https://www.wireshark.org/. Accessed 11 Nov 2016

23. Open vSwitch. http://openvswitch.org/. Accessed 18 Oct 2016

24. Click Modular Router Project (2009). http://www.read.cs.ucla.edu/click/click. Accessed 11 Nov 2016

\section{Submit your manuscript to a SpringerOpen ${ }^{\circ}$ journal and benefit from:}

- Convenient online submission

- Rigorous peer review

- Immediate publication on acceptance

- Open access: articles freely available online

- High visibility within the field

- Retaining the copyright to your article

Submit your next manuscript at $\boldsymbol{s p r i n g e r o p e n . c o m ~}$ 